\title{
Urethral Clear Cell Adenocarcinoma
}

National Cancer Institute

\section{Source}

National Cancer Institute. Urethral Clear Cell Adenocarcinoma. NCI Thesaurus. Code C6172.

A morphologic variant of urethral adenocarcinoma characterized by the presence of tubulocystic or papillary structures lined with clear cuboidal or hobnail cells. 